\title{
Segurança dos alimentos: influência sazonal \\ na contaminação parasitária em alface (Lactuca sativa L.) comercializada em feiras livres de Belém, Pará
}

\section{Food safety: seasonal influence on parasitic contamination of lettuce (Lactuca sativa $L$.) commercialized in street markets in Belém, Pará, Brazil}

\author{
Fernanda Alencar Medeiros ${ }^{1}$, Tatiane Rodrigues de Oliveira², \\ Sergio Marcelo Rodríguez Málaga ${ }^{1 *}$ (i) \\ ${ }^{1}$ Universidade Federal do Pará (UFPA), Instituto de Ciências Biológicas, Laboratório de Parasitologia, Belém/PA - \\ Brasil \\ ${ }^{2}$ Faculdade Joaquim Nabuco, Laboratório de Parasitologia, Fortaleza/CE - Brasil \\ *Corresponding Author: Sergio Marcelo Rodríguez Málaga, Universidade Federal do Pará (UFPA), Instituto de \\ Ciências Biológicas, Laboratório de Parasitologia, Rua Augusto Corrêa, 01, sala 324, CEP: 66075-110, Belém/PA - \\ Brasil, e-mail: sergiorm@ufpa.br
}

Cite as: Medeiros, F. A., Oliveira, T. R., \& Málaga, S. M. R. (2019). Food safety: seasonal influence on parasitic contamination of lettuce (Lactuca sativa L.) commercialized in street markets in Belém, Pará, Brazil. Brazilian Journal of Food Technology, 22, e2018205. https://doi.org/10.1590/1981-6723.20518

\begin{abstract}
Resumo
O consumo de vegetais in natura no Brasil e no mundo vem apresentando um crescimento significativo. Dentre estes alimentos, a alface crespa (Lactuca sativa L.) é a hortaliça folhosa de maior consumo no país, pois se adequa a diversos tipos de dieta e apresenta baixo valor calórico. Entretanto, a preferência por este tipo de alimentos e seus benefícios pode expor os consumidores ao risco de contrair infecções por diversos patógenos. O presente estudo objetivou avaliar a influência da sazonalidade no grau de contaminação parasitária em alfaces crespas comercializadas em quatro feiras livres do município de Belém-PA. Um total de 120 exemplares de alfaces foram adquiridas aleatoriamente nas feiras municipais de Belém, durante a estação seca e chuvosa, as quais foram processadas e analisadas individualmente pelo método de sedimentação espontânea. Observou-se que o nível de contaminação da hortaliça foi similar nos distintos períodos sazonais, sendo de $36,7 \%$ durante o período de seca e $38,7 \%$ na estação chuvosa. A contaminação parasitária mista, detectada especialmente no período chuvoso, sugere que os índices pluviométricos podem influenciar no aumento do número de espécies de parasitos que potencialmente podem afetar a população. Os resultados demonstraram uma elevada contaminação por agentes parasitários em todas as feiras que comercializavam a hortaliça. As principais estruturas parasitárias detectadas foram ovos de Ascaris sp., Ancylostoma sp., Strongyloides sp., Enterobius vermiculares, Taenia sp., oocisto de Toxoplasma gondii, além de diversas sujidades. $O$ estudo identificou uma taxa moderada de contaminação por helmintos e protozoários de alfaces
\end{abstract}


comercializadas nas feiras livres no município de Belém, evidenciando o contínuo risco de infecção parasitária humana através do consumo de hortaliças cruas, o que justifica a implementação de medidas que propiciem uma melhora na qualidade higiênico-sanitária.

Palavras-chave: Hortaliça; Estações seca e chuvosa; Helmintos; Protozoário; Cultura convencional; Contaminação alimentar.

\section{Abstract}

The consumption of fresh vegetables in Brazil and in the world has been increasing significantly. Of these foods, lettuce (Lactuca sativa L.) is the vegetable most consumed in Brazil, since it is suitable for several types of diet and presents a low caloric value. Nevertheless, the preference for this type of food and its benefits may expose consumers to the risk of contracting infections due to various pathogens. The objective of the present study was to evaluate the influence of seasonality on the parasitic contamination of lettuces commercialized in four street markets in the city of Belém-PA, Brazil. One hundred and twenty lettuce specimens were obtained from municipal street markets in Belém during the dry and rainy seasons, and then processed and analysed individually by the spontaneous sedimentation method. The level of contamination of the vegetables was found to be similar in the different seasonal periods, being $36.7 \%$ during the dry season and $38.7 \%$ in the rainy season. Mixed parasitic contamination, detected especially in the rainy season, suggests that the rainfall indices may influence the increase in the number of parasite species potentially affecting the population. The results showed elevated contamination by parasitic agents in all the street markets that commercialized the vegetable. The main parasitic species detected were Ascaris sp., Ancylostoma sp., Strongyloides sp., Enterobius vermicularis, Taenia sp., Toxoplasma gondii oocyst, and other dirt. The study identified a moderate rate of contamination by helminths and protozoans in the lettuces sold in the street markets of the city of Belém, showing evidence of the continuous risk of parasitic infections to humans from the consumption of raw vegetables, which justifies the implementation of measures that allow for an improvement in the hygienic-sanitary quality.

Keywords: Vegetables; Dry and rainy seasons; Helminths; Protozoans; Conventional cultivation; Food contamination.

\section{Introdução}

Os vegetais são parte essencial de uma dieta saudável, devido ao seu valor nutricional, pois são fontes de vitaminas, elementos minerais e fibras (Mohamed et al., 2016). Além disso, o aumento de evidências científicas que apontam o consumo regular de vegetais na redução de doenças crônicas, como diabetes, doenças cardíacas, câncer e obesidade, tem contribuído para o aumento exponencial do consumo de vegetais in natura no mundo (Padmanandan et al., 2016). Estes aspectos favorecem o consumo de hortaliças na sua forma crua, podendo servir como uma importante fonte de transmissão de infecções parasitárias (Eraky et al., 2014).

As condições sanitárias envolvidas nas etapas de produção dos vegetais que incluem a contaminação do solo com resíduos animais e humanos, a aplicação de adubo compostado, a irrigação com água de mananciais ou remansos que recebem descargas de dejetos de forma direta ou arrastados pela chuva, e o contato com animais, como aves, moscas e ratos, desempenham um papel importante no processo de difusão das enteroparasitoses (Balarak et al., 2016; Maciel et al., 2014).

Entre as hortaliças, a alface (Lactuca sativa L.) é amplamente consumida na forma de salada pela população mundial, tendo especial importância para a saúde pública, pois serve como uma importante via de transmissão de cistos e oocistos de protozoários, e ovos/larvas de helmintos, relatados em inquéritos de diferentes partes do mundo, como Sudão (Mohamed et al., 2016), Colômbia (Polo et al., 2016); Egito (Ahmad et al., 2016; Eraky et al., 2014), Brasil (Silverio et al., 2014; Silva et al., 2015; Mesquita et al., 2015), entre outros. 
Considerado um vegetal de grande importância do ponto de vista econômico, o segmento de alface crespa no Brasil vem liderando a comercialização nas últimas décadas, motivado, principalmente, pelo ciclo precoce de desenvolvimento, podendo atingir seu ponto de colheita com até 30 dias após o transplante. Note-se que a alface crespa possui uma boa adequação ao cultivo no verão, em regiões de altas temperaturas, minimizando as perdas que havia com a alface lisa e o cultivo no sistema tradicional (Sala \& Costa, 2012).

Do ponto de vista da segurança e qualidade dos alimentos, as enteroparasitoses se enquadram na categoria de doenças veiculadas por alimentos e representam um grave problema de saúde pública, causando uma gama de patologias que podem ter consequências devastadoras para os indivíduos e/ou comunidade acometida (Petney et al., 2016). A Organização Mundial da Saúde estima que cerca de 1,5 bilhão de pessoas, correspondente a $24 \%$ da população mundial, são afetadas por infecções parasitárias intestinais (World Health Organization, 2016).

A notificação não obrigatória de casos isolados para a maioria das enteroparasitoses, bem como a ausência de ações integradas no controle, como o diagnóstico de rotina de parasitos, têm acarretado uma subnotificação de casos, o que dificulta o dimensionamento do problema e o planejamento de ações por parte dos órgãos competentes. Além disso, dados do Instituto Brasileiro de Geografia e Estatística (2011) apontam que as regiões Norte e Nordeste do país apresentam um crescimento absoluto da população que não é acompanhado pelas melhorias do esgotamento sanitário e do acesso à água tratada, fato que pode acarretar um aumento das infecções parasitárias.

Neste contexto, o conhecimento das condições e dos fatores que influenciam a contaminação por enteroparasitos em hortaliças é essencial para uma correta avaliação do risco de infecção, o que permitiria a promoção de educação sanitária de caráter preventivo, que consiga assegurar a qualidade do produto, resultando na redução da transmissão dessas doenças (Landivar \& Vidigal, 2015).

Assim, o objetivo do presente estudo foi avaliar a influência da sazonalidade no grau de contaminação parasitária em alfaces crespas (Lactuca sativa L.) comercializadas em feiras livres do município de Belém, estado do Pará.

\section{Material e métodos}

A pesquisa se classifica como um estudo transversal de abordagem quantitativa e foi realizada durante as estações de seca da região, entre os meses de setembro e novembro de 2014, e a estação chuvosa, nos meses de fevereiro a maio de 2015. Um total de 120 amostras de alface crespa (Lactuca sativa L.), originadas de culturas convencionais e produzidas no município de Belém, foram adquiridas de forma aleatória em quatro feiras livres da cidade (Feira A - zona sul, Feira B - zona centro-sul, Feira C - zona oeste e Feira D - zona centro-sul). De cada estabelecimento, foram adquiridas 15 unidades da hortaliça, completando 60 unidades na estação seca e 60 na estação chuvosa.

As amostras de alfaces foram acondicionadas, individualmente, em sacos plásticos estéreis, identificadas e transportadas ao Laboratório de Parasitologia Humana, no Instituto de Ciências Biológicas, na Universidade Federal do Pará (UFPA), para a análise parasitológica.

No laboratório, as amostras foram processadas individualmente, empregando-se a técnica de sedimentação espontânea (Hoffman et al., 1934). Em resumo, aproximadamente $100 \mathrm{~g}$ de folhas de alface de cada amostra foram pesadas em balança semianalítica (Ohaus LS2000) e submersas em $250 \mathrm{~mL}$ de água destilada para o processo de lavagem por fricção manual, com o intuito de desprender as possíveis estruturas parasitárias. O líquido resultante da lavagem foi filtrado utilizando filtro parasitológico e gaze, sendo posteriormente transferido para cálices de sedimentação espontânea, decantando por um período de duas horas.

Após este período, o sobrenadante foi removido e $500 \mu \mathrm{L}$ do precipitado foram transferidos para tubos plásticos estéreis de $1,5 \mathrm{~mL}$, os quais foram mantidos em refrigeração a $4{ }^{\circ} \mathrm{C}$ até análise microscópica. Para 
a observação microscópica, $50 \mu \mathrm{L}$ de cada amostra foram analisados por três observadores independentes, em microscópio Olympus CX40 com objetivas de 20× e 40×.

\section{Resultados e discussão}

As doenças transmitidas por alimentos são uma das principais causas de morbidade em todo o mundo. As hortaliças, especialmente as consumidas cruas, são amplamente recomendadas como parte da alimentação diária, entretanto têm particular importância em saúde pública, sendo uma das principais vias de transmissão parasitária.

Diversos estudos apontam uma alta prevalência de estágios infecciosos parasitários em hortaliças vendidas em feiras livres e mercados, especialmente nos países em desenvolvimento, onde o saneamento e a higiene ambiental são precários (Maciel et al., 2014; Luz et al., 2014; Chau et al., 2014; Eraky et al., 2014). Entretanto, poucos estudos caracterizam o efeito da sazonalidade na incidência de enteroparasitos em hortaliças comercializadas (Silverio et al., 2014).

No presente estudo, do total de 120 amostras de alfaces analisadas procedentes das feiras livres do município de Belém-PA, 36,7\% (22 amostras) das alfaces obtidas durante o período de estiagem e 38,3\% (23 amostras) obtidas no período chuvoso apresentaram contaminação por estruturas parasitárias, sugerindo que os índices pluviométricos da região não influenciam no índice total de parasitismo da hortaliça, porém demonstram uma taxa moderada de contaminação por helmintos e protozoários.

O resultado obtido difere consideravelmente do encontrado por Uga et al. (2009), que demonstraram um percentual elevado de ovos de helmintos em amostras de vegetais provenientes de Hanói, no Vietnã, durante o período de seca (78\%), quando comparado à estação chuvosa (22\%). É importante reforçar que a variação da prevalência pode ser atribuída a vários fatores, como localização geográfica, hábitos higiênicos relacionados à população, condições climáticas, bem como tipo de água utilizada na irrigação e métodos de manuseio pós-colheita (Eraky et al., 2014).

Na literatura, o percentual de positividade por enteroparasitos em amostras de alfaces apresenta taxas variadas. No estudo realizado com vegetais frescos comercializados nas feiras livres do estado de Khartoum, Sudão, 36,4\% das amostras de alfaces estavam contaminadas por espécies parasitárias de importância médica (Mohamed et al., 2016). Por outro lado, o estudo realizado no município de São Mateus, Espirito Santo, demonstrou que $86,8 \%$ das amostras de alfaces obtidas em estabelecimentos comerciais estavam contaminadas (Brauer et al., 2016).

Segundo Martins et al. (2014), as variáveis ambientais e as modificações introduzidas pelas atividades humanas alteram a frequência de infecções por helmintos, pois propicia viabilidade destas formas parasitárias através da criação de microambientes favoráveis.

Neste estudo, todas as amostras positivas obtidas no período de estiagem apresentaram monoparasitismo, entretanto foi observado, nas análises positivas obtidas no período chuvoso, o poliparasitismo, sendo detectado prevalentemente ovos de Ancylostoma sp., Ascaris sp. e de Strongyloides sp. É possível que os percentuais de contaminação mista observados na época chuvosa estejam associados ao acúmulo de água nos locais de exposição ou ao armazenamento das alfaces, corroborando com o estudo de Simões et al. (2001), que relatam que a contaminação de hortaliças por parasitos sofre influências sazonais, com índices maiores na estação chuvosa.

Fatores, como alta umidade, temperatura entre $20{ }^{\circ} \mathrm{C}$ e $30{ }^{\circ} \mathrm{C}$, e boa oxigenação, são condições indispensáveis para o desenvolvimento de ovos de helmintos, favorecendo os processos de embriogênese, formação da larva e eclosão (Taylor et al., 2017). Os aspectos climáticos citados acima são característicos da região do estudo, que apresenta uma homogeneidade sazonal de temperatura e umidade do ar. A cidade de 
Belém caracteriza-se por apresentar temperatura anual e umidade do ar média em torno de $26{ }^{\circ} \mathrm{C}$ e $84 \%$, respectivamente, e precipitação pluvial média entre 3.000 e $4.000 \mathrm{~mm}$ (Costa \& Blanco, 2018).

Quanto ao percentual de contaminação por estabelecimento estudado, nota-se que a positividade das amostras durante o período de estiagem variou de um mínimo de 26,3\% ao máximo de 53,3\%. Por outro lado, o percentual de amostras de alfaces contaminadas durante o período chuvoso por estabelecimento variou entre $13 \%$ e $60 \%$. Todos os estabelecimentos estudados apresentaram algum grau de contaminação, porém a feira que apresentou maior taxa de contaminação por enteroparasitos, independentemente do período avaliado, foi a Feira B (Figura 1).

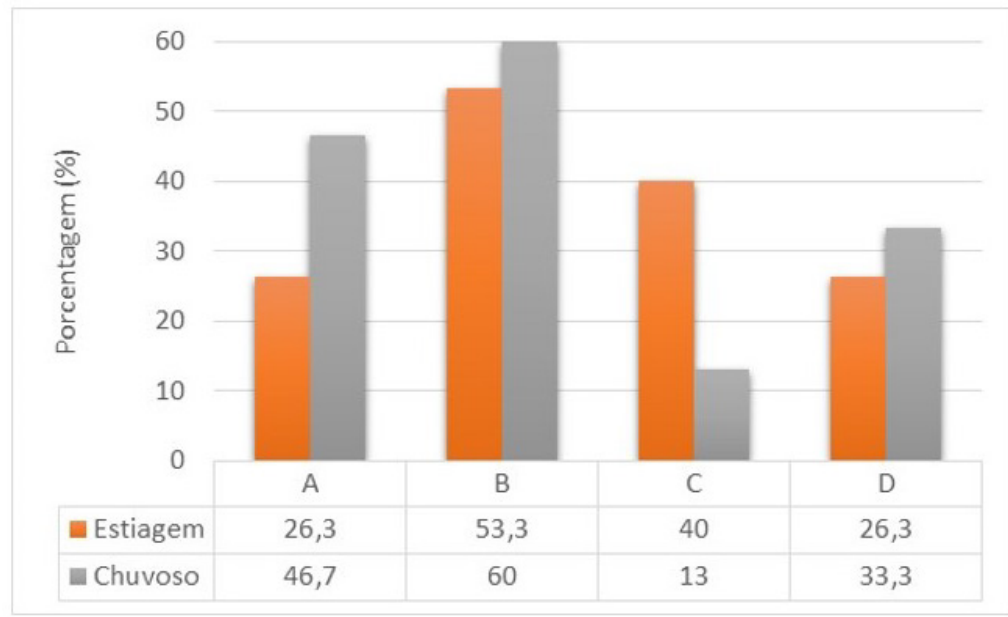

Figura 1. Análise comparativa da frequência de parasitos encontrados em amostras de alfaces, segundo os locais de coletas durante os períodos chuvoso e de estiagem.

A Figura 2 apresenta o percentual de enteroparasitos encontrados nas amostras de alfaces em ambos períodos analisados, sendo prevalentes os ovos de Ancylostoma sp. e Ascaris sp., e os menos frequentes, Enterobius vermiculares e oocisto de Toxoplasma gondii. Os gêneros Ascaris e Ancylostoma são dos mais frequentes do mundo, com ampla distribuição geográfica, com frequência variável em virtude das condições climáticas e ambientais, estando associados diretamente com o baixo grau de desenvolvimento socioeconômico da população (Moura et al., 2016).

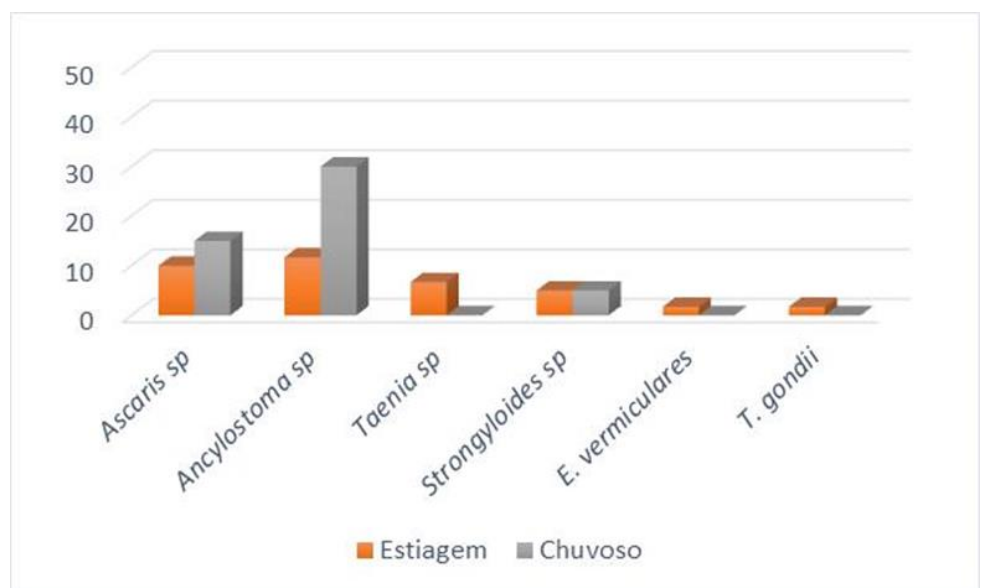

Figura 2. Distribuição das estruturas parasitárias presentes nas amostras de hortaliças comercializadas no município de Belém, nos períodos de estiagem e chuvoso. 
A Figura 3 mostra imagens de estruturas parasitárias encontradas neste estudo, tais como ovos de Ascaris sp., Taenia sp. e oocisto de Toxoplasma gondii. As espécies de helmintos e protozoários detectadas nas amostras de alfaces em Belém-PA também foram observadas em estudos realizados em diferentes regiões do país e indicam que as condições higiênico-sanitárias de cultivo e comercialização das hortaliças não diferem entre as regiões.
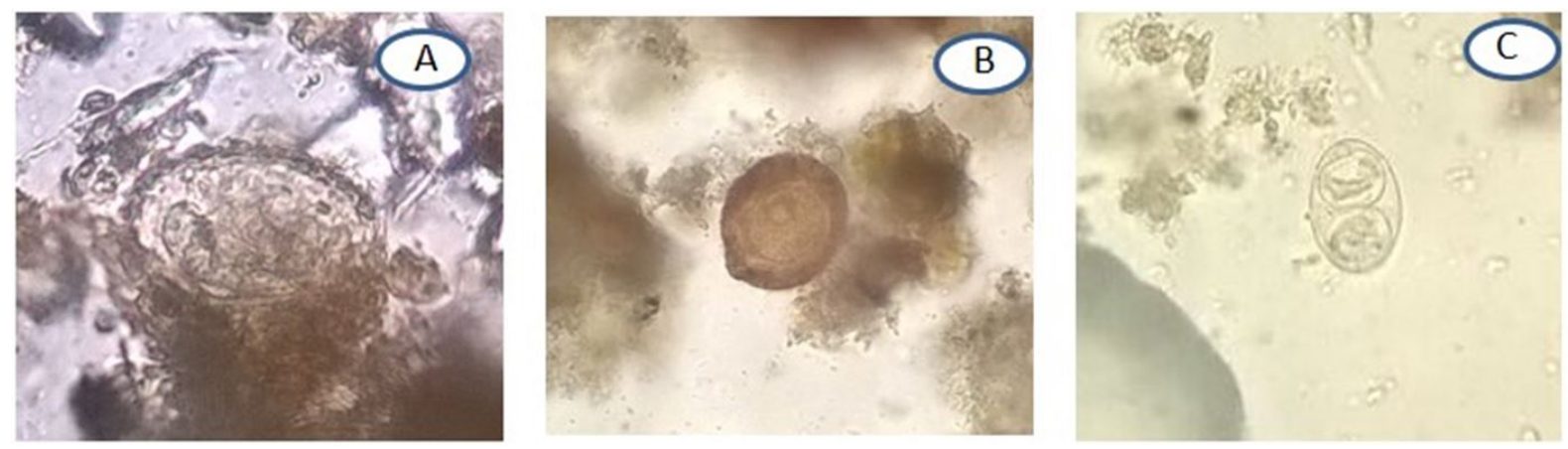

Figura 3. Estruturas parasitárias detectadas em alfaces comercializadas nas feiras livres do município de Belém.

(A) Ovos de Ascaris sp.; (B) Ovos de Taenia sp.; (C) Oocisto de Toxoplasma gondii.

Maciel et al. (2014) demonstraram que alfaces obtidas em feiras livres no Distrito Federal estavam contaminadas principalmente por helmintos (ancilostomídeos e Strongyloides sp.) e protozoários (Entamoeba sp.). Em estudo realizado em Teresina, Piauí, foi demonstrado que as amostras de alfaces estavam contaminadas principalmente por ancilostomídeos, Ascaris sp. e Strongyloides sp., resultado semelhante ao observado neste estudo (Mesquita et al., 2015). De forma similar, Melo et al. (2017) identificaram a contaminação de hortaliças minimamente processadas provenientes de supermercados de Fortaleza, Ceará por protozoários, como T. gondii ( $8,3 \%)$, entre outros, e helmintos, como ovos e larvas de ancilostomídeos (41,7\%), larvas de Strongyloides sp. (33,3\%) e ovos de Taenia sp. (16,7\%).

Portanto, a contaminação parasitária presente nas amostras de alfaces comercializadas nas feiras livres do município de Belém, evidenciada neste estudo, demonstra a qualidade insatisfatória do produto para o consumo humano, alertando sobre o risco da aquisição de infecções por enteroparasitos.

\section{Conclusões}

Conclui-se, a partir da análise dos dados obtidos neste estudo, a necessidade de um sistema atuante de vigilância sanitária, com foco no cumprimento das normas operacionais por parte dos produtores e comerciantes de hortaliças do município, garantindo assim um alimento seguro ao consumidor. A adoção de medidas de conscientização coletiva, visando à aplicação de boas práticas de produção e comercialização, pode propiciar uma melhoria na qualidade desses produtos.

\section{Agradecimentos}

Os autores agradecem ao Conselho Nacional de Desenvolvimento Científico e Tecnológico (CNPq) e à Universidade Federal do Pará pela concessão da bolsa de estudo.

\section{Referências}

Ahmad, S. O., El Fadaly, H. A., Zaki, M. S., \& Barakat, A. M. A. (2016). Incidence of zoonotic parasites in Egyptian raw vegetable salads. Life Science Journal, 13(2), 27-31. 
Balarak, D., Ebrahimi, M., Modrek, M. J., Bazrafshan, E., Mahvi, A. H., \& Mahdavi, Y. (2016). Investigation of parasitic contaminations of vegetables sold in markets in the city of tabriz in 2014. Global Journal of Health Science, 8(10), 178-184. PMid:27302434. http://dx.doi.org/10.5539/gjhs.v8n10p178

Brauer, A. M. N. W., Silva, J. C., \& Souza, M. A. A. (2016). Distribuição de enteroparasitos em verduras do comércio alimentício do município de São Mateus, Espírito Santo, Brasil. Natureza on line, 14(1), 55-60. Recuperado em 27 de agosto de 2018, de http://www.naturezaonline.com.br/natureza/conteudo/pdf/NOL20151101.pdf

Chau, H. L. Q., Thong, H. T., Chao, N. V., Hung, P. H. S., Van Hai, V., Van An, L., Fujieda, A., Ueru, T., \& Akamatsu, M. (2014). Microbial and parasitic contamination on fresh vegetables sold in traditional markets in Hue City, Vietnam. Journal of Food and Nutrition Research, 2(12), 959-964. http://dx.doi.org/10.12691/ffnr-2-12-16

Costa, C. E. A. S., \& Blanco, C. J. C. (2018). Influência da variabilidade climática sobre a erosividade em Belém (PA). Revista Brasileira de Meteorologia, 33(3), 509-520. http://dx.doi.org/10.1590/0102-7786333010

Eraky, M. A., Rashed, S. M., Nasr, M.-S., El-Hamshary, A. M., \& Salah El-Ghannam, A. (2014). Parasitic contamination of commonly consumed fresh leafy vegetables in Benha, Egypt. Journal of Parasitology Research, 2014, 613960. PMid:25024845. http://dx.doi.org/10.1155/2014/613960

Hoffman, W. A., Pons, J. A., \& Janer, J. L. (1934). The sedimentation concentration method in Schistosomiasis mansoni. The Puerto Rico Journal of Public Health and Tropical Medicine, 9, 283-298.

Instituto Brasileiro de Geografia e Estatística - IBGE. (2011). Atlas de saneamento 2011. Rio de Janeiro.

Landivar, E., \& Vidigal, T. (2015). Avaliação parasitológica de alfaces crespas comercializadas em feiras e supermercados no município de São Miguel do Oeste, SC. Unoesc \& Ciência: ACBS, 6(1), 29-36. Recuperado em 27 de agosto de 2018, de https://editora.unoesc.edu.br/index.php/acbs/article/view/6655/pdf_77

Luz, J. R. D., Câmara, H. C. F., Lima, D. V. P., Silva, M. H. R., Costa, E. L., \& Zelenoy, C. K. G. (2014). Avaliação da contaminação parasitária em alfaces (Lactuca sativa) comercializadas em feiras livres na região da Grande Natal, Rio Grande do Norte. Nutrivisa, 1(2), 16-19.

Maciel, D., Gurgel-Gonçalves, R., \& Machado, E. R. (2014). Ocorrência de parasitos intestinais em hortaliças comercializadas em feiras no Distrito Federal, Brasil. Revista de Patologia Tropical, 43(3), 351-359. http://dx.doi.org/10.5216/rpt.v43i3.32216

Martins, A. S., Duarte, A. N., Carvajal, E., Sarquis, M. I. M., \& Fernandes, O. C. C. (2014). Controle da qualidade microbiológica e parasitária em áreas de recreação. Revista Eletrônica Gestão \& Saúde, 5(3), 2059-2078.

Melo, M. V. C., Pacheco, D. T. L., Lustosa, I. B. S., Rodriguez-Malága, S. M., \& Oliveira, T. R. (2017). Presencia de formas infectivas de parásitos intestinales en hortalizas mínimamente procesadas, comercializadas en el municipio Fortaleza, CearáBrasil. Parasitología Latinoamericana, 66(2), 5-12. Recuperado em 27 de agosto de 2018, de http://sociedadchilenaparasitologia.cl/wp-content/uploads/2018/04/PLA-66-2.pdf

Mesquita, D. R., Silva, J. P., Monte, N. D. P., Sousa, R. L. T., Silva, R. V. S., Oliveira, S. S., Leal, A. R. S., \& Freire, S. M. (2015). Ocorrência de parasitos em alface-crespa (Lactuca sativa L.) em hortas comunitárias de Teresina, Piauí, Brasil. Revista de Patologia Tropical, 44(1), 67-76. http://dx.doi.org/10.5216/rpt.v44i1.34802

Mohamed, M. A., Siddig, E. E., Elaagip, A. H., Edris, A. M. M., \& Nasr, A. A. (2016). Parasitic contamination of fresh vegetables sold at central markets in Khartoum state, Sudan. Annals of Clinical Microbiology and Antimicrobials, 15(17), 1-7. PMid:26968696. http://dx.doi.org/10.1186/s12941-016-0133-5

Moura, L. R., Santos, T., \& Viegas, A. A. (2016). Avaliação parasitológica em Lactuca sativa (alface) e Brassica oleracea L. (couve) procedentes da Ceasa no município de Anápolis - GO. Revista Educação em Saúde, 4(1), 59-66. Recuperado em 27 de agosto de 2018, de http://periodicos.unievangelica.edu.br/index.php/educacaoemsaude/article/view/1696

Padmanandan, A., Singh, S., \& Gaind, R. (2016). Parasitic contamination in commonly consumed raw vegetables: A review study. Epidemiology International, 1(1), 1-4. Recuperado em 27 de agosto de 2018, de https://medical.adrpublications.in/index.php/Journal-EpidemInternational/article/view/654/521

Petney, T. N., Sithithaworn, P., Andrews, R. H., \& Webster, J. P. (2016). Foodborne trematodes: A diverse and challenging group of neglected parasites. Transactions of the Royal Society of Tropical Medicine and Hygiene, 110(1), 1-3. PMid:26740355 http://dx.doi.org/10.1093/trstmh/trv112

Polo, G. A., Benavides, C. J., Astaiza, J. M., Vallejo, D. A., \& Betancourt, P. (2016). Determinación de enteroparásitos en Lactuca sativa en fincas dedicadas a su producción en Pasto, Colombia. Biomédica, 36(4), 525-534. PMid:27992979. http://dx.doi.org/10.7705/biomedica.v36i4.2914

Sala, F. C., \& Costa, C. P. (2012). Retrospectiva e tendência da alfacicultura brasileira. Horticultura Brasileira, 30(2), 187-194 http://dx.doi.org/10.1590/S0102-05362012000200002

Silva, M. R. P., Pinheiro, F. C., De Paula, M. T., \& Prigol, M. (2015). Avaliação parasitológica de alfaces (Lactuca sativa) comercializadas em um município da Fronteira Oeste, Rio Grande do Sul. Revista de Patologia Tropical, 44(2), 163-169. http://dx.doi.org/10.5216/rpt.v44i2.36646

Silverio, N. M., Resende, R. A., Pessoa, R. S., \& França-Botelho, A. C. (2014). Interferências sazonais na contaminação parasitológica de alfaces em Araxá (MG), Brasil. Revista Saúde e Pesquisa, 7(3), 389-394. Recuperado em 27 de agosto de 2018, de http://periodicos.unicesumar.edu.br/index.php/saudpesq/article/view/3501/2478

Simões, M., Pisani, B., Marques, E. G. L., Prandi, M. A. G., Martini, M. H., Chiarini, P. F. T., Antunes, J. L. F., \& Nogueira, A. P. (2001). Hygienic-sanitary conditions of vegetable and irrigation water from kitchen gardens in the municipality of Campinas, SP. Brazilian Journal of Microbiology, 32(4), 331-333. http://dx.doi.org/10.1590/S1517-83822001000400015 
Segurança dos alimentos: influência sazonal na contaminação parasitária em alface (Lactuca sativa L.) comercializada em feiras livres de Belém, Pará

Medeiros, F. A. et al.

Taylor, M. A., Coop, R. L., \& Wall, R. L. (2017). Parasitologia veterinária (4. ed.). Rio de Janeiro: Guanabara Koogan.

Uga, S., Hoa, N. T. V., Noda, S., Moji, K., Cong, L., Aoki, Y., Rai, S. K., \& Fujimaki, Y. (2009). Parasite egg contamination of vegetables from a suburban market in Hanoi, Vietnam. Nepal Medical College Journal, 11(2), 75-78. PMid:19968142.

World Health Organization - WHO. (2016). Soil-transmitted helminth infections. Geneva: WHO. Recuperado em 27 de agosto de 2018, de http://www.who.int/mediacentre/factsheets/fs366/es

Financiamento: Nenhum. 